\title{
Desvendando a medição nos ensaios químicos: 1. A curva analíitca ou de calibração
}

\author{
Oscar Bahia Filho ${ }^{1, *}$, Patrícia Regina Prada², \\ Carla Meneghesso², Fernando Mauro Lanças ${ }^{2}$ \\ ${ }^{1}$ TQW Consultoria em Quimica e Qualidade \\ e-mail: oscarbfilho@terra.com.br \\ ${ }^{2}$ Instituto de Química de São Carlos, Universidade de São Paulo \\ Cep 13560-970, São Carlos, SP, Brasil
}

\section{Resumo}

Nos ensaios químicos, o modelo matemático utilizado como base de quantificação é o responsável pela transformação de uma unidade de medida na unidade de quantificação de interesse. Por tratar de uma série de operações, a possibilidade de propagação de erros é preocupante e, nesse sentido, a confiabilidade de todas essas operações deve ser estabelecida e conhecida. O presente trabalho, o primeiro de uma série, visa discutir a contribuição da etapa de construção da curva analítica ou de calibração no processo do ensaio com base na sua estimativa da incerteza conforme preconizado pelas diretrizes das normas ISO e BPL. A aplicação desses conceitos culminou na elaboração de um procedimento operacional e foi aplicado na determinação de fluazuron em tecidos animais por cromatografia líquida acoplada à espectrometria de massas em tandem (LC-MS/MS).

\section{Palavras-chave}

Estimativa da incerteza; curva de calibração ou analítica; HPLC; sistema de gestão da qualidade; ISO 17025; BPL.

\section{Unraveling measurement in chemical analysis: 1. The analytical or calibration curve}

Abstract

In a typical quantitative chemical analysis, the mathematical model used for the quantification process is responsible for the transformation of a measured unit into the quantity of interest. Once dealing with a series of operations, the possibility of propagation of errors is a real concern; the trustworthiness of all these operations must be well established and be known. The present work, the first one of a series, aims to present and discuss the contribution of the stage of construction of the analytical or calibration curve in the process of the assay by estimating its uncertainty based upon the ISO 17025 and GLP guidelines. The application of these concepts culminated in the elaboration of an operational procedure that was applied to the determination of fluazuron in animal tissues using liquid chromatography coupled to mass spectrometer (LC-MS/MS).

\section{Keywords}

Measurement uncertainty; analytical or calibration curve; HPLC; LC-MS/MS; quality system management; ISO 17025; GLP guidelines. 


\section{Introdução}

A internacionalização do mercado, aliada à adoção crescente das regras de mercado justo, trouxeram para as empresas de produtos e de serviços dois importantes parâmetros: a comparabilidade e a rastreabilidade ${ }^{[1,2]}$. Ambos os termos, hoje bastante comuns, fazem parte de um trabalho sistemático e organizado, geralmente controlado por um sistema que busca constância e diferenciação no que a organização produz ou oferece. A manutenção da constância dos produtos ou serviços está associada à ideia de garantia da qualidade resultante da aplicação do sistema de gestão, denominado sistema de gestão da qualidade (SGQ). Já a diferenciação está associada à conquista ou domínio de segmento do mercado.

Como os negócios são quase sempre baseados em quantificação, a confiabilidade da medição é um ponto nevrálgico. Desta forma, em algum momento do processo, deve haver pelo menos uma etapa responsável pela medida de uma determinada característica do produto ou serviço que caracteriza sua especificação antes da sua comercialização. Tal etapa é denominada de controle da qualidade. Para assegurar que todas as atividades deste controle sejam executadas em conformidade com procedimentos definidos, são realizadas ações que garantam a sua qualidade. Via de regra, a etapa do controle da qualidade é feita utilizando equipamentos que são responsáveis pelas medições inerentes; consequentemente, para fins de comparabilidade, este deve estar calibrado em relação a um padrão de referência.

A garantia da confiabilidade da medição é baseada em quatro parâmetros: (i) procedimentos operacionais padrão (POPs ou SOPs), que devem ser conduzidos por (ii) pessoal habilitado utilizando (iii) equipamentos calibrados instalados ou mantidos em (iv) local e condi- ções ambientais adequadas ${ }^{[3]}$. Como todo SGQ é elaborado para atender a critérios específicos de um escopo, ou faixa de cobertura, as organizações utilizam as diretrizes das normas de gestão ABNT NBR ISO/IEC $17025^{[4]}$, ABNT NBR ISO $9001^{[5]} \mathrm{e}$ das Boas Práticas de Laboratório ${ }^{[6]}$ (BPL ou GLP) para estabelecer a confiabilidade e adequabilidade de seus produtos ou serviços, no que se refere ao item equipamentos.

Embora as diretrizes destas diferentes normas sejam direcionadas a aspectos diferentes da conformidade da qualidade, a calibração, nas três, é citada de maneira a atender a cada uma delas. Enquanto a ISO 17025 tem foco no cliente e é usada para atestar a competência da empresa em realizar ensaios em escopos definidos, a ISO 9001, também com foco no cliente, é usada para garantir a conformidade de um produto. Já a BPL visa atender à legislação pertinente, e é usada para descrever a condução de estudos específicos. Para a ISO 17025, este assunto é tratado no item 5.5; já para a ISO 9001 o mesmo assunto é apresentado no item 7.6 e na BPL no item 4.

O elemento da norma ISO 17025 no item 5.5, de forma geral, estabelece que devem existir programas de calibração para os instrumentos críticos antes de serem colocados em uso e que o certificado de calibração deve ser avaliado criticamente quanto à tolerância do instrumento na faixa especificada. Quando em uso, se um determinado equipamento produzir resultados de ensaios suspeitos ou fora das especificações, este deverá ser claramente marcado como fora de serviço até que a causa do desvio seja identificada e seja demonstrado por meio de calibração ou verificação que o equipamento encontra-se em condições adequadas de uso. No caso em que um equipamento saia do controle direto da organização, verificações devem ser utilizadas para garantir que o equipamento continue funcionando de maneira correta. 
A ISO 9001 define, no item 7.6, que, para assegurar resultados válidos, os equipamentos devem ser calibrados e/ou verificados de forma periódica ou antes do uso. Recomenda que estas calibrações/verificações sejam feitas contra padrões de medição rastreáveis a padrões internacionais, mas permite o uso de padrões não rastreáveis, desde que não exista um padrão de medição de referência e seja registrada qual foi a base de comparação utilizada. É necessário manter os registros dos resultados de calibração e verificação bem como a avaliação destes dados, que devem ser analisados conforme critérios previamente definidos. A organização deve ter, ainda, mecanismos para identificar rapidamente a necessidade de ajuste ou reajuste no equipamento, para que possa tomar as ações apropriadas.

Já as diretrizes preconizadas pelo documento normativo BPL trata a calibração de forma mais abrangente que os documentos ISO. A norma NIT-DICLA-035, que rege os Princípios das Boas Práticas de Laboratório, estabelece que os equipamentos utilizados em um estudo devem ser periodicamente inspecionados, ter programas de manutenção e calibração de acordo com procedimentos, e os registros destas atividades devem ser mantidos. A preocupação desta abordagem é impedir que equipamentos críticos não calibrados interfiram adversamente com os sistemas teste.

O termo comum nas três normas é que todos os equipamentos críticos devem ser calibrados e periodicamente verificados antes dos ensaios. Contudo, não está claro o período entre as calibrações, nem os mecanismos de verificação periódica da calibração. Entretanto, para assegurar a confiabilidade da medida, a calibração dos equipamentos críticos é apenas um dos parâmetros, embora seja o parâmetro de partida. Assim, se pode tomar a seguinte premissa: toda medida obtida com equipamentos calibrados tem maior probabilidade de ser confiável, enquanto que, se essa for feita em equipamentos não calibrados, a medida dificilmente será confiável. Assim, o ponto de partida para uma medida confiável e comparável é utilizar equipamentos calibrados. Aqui cabe salientar que se pode dividir as medidas em dois grupos ${ }^{[7]}$ : no primeiro, as medições são diretas, isto é, o resultado do equipamento é dado na mesma unidade da medida do mensurando, por exemplo, a medida da espessura de peça utilizando um paquímetro. No segundo, as medidas são indiretas, ou seja, o resultado do equipamento deve ser transformado para a unidade de interesse, por exemplo, o teor de ferro em uma formulação farmacêutica utilizando um procedimento de mineralização do comprimido e, posteriormente, a medida em um espectrômetro de absorção atômica. Este último é representado pela quase totalidade dos ensaios químicos.

Como a medição nos ensaios químicos é indireta, a transformação da medida do equipamento na unidade de interesse do mensurando é feita utilizando uma comparação direta de uma série de soluções padrão de concentração conhecidas contra os valores medidos, seguida de transformações matemáticas até a obtenção do resultado final. A essa relação estabelecida, se dá o nome de curva de calibração ou curva analítica. Entretanto, o equipamento utilizado para estabelecer esta relação deve, segundo as diretrizes das normas, ser calibrado. Assim, para garantir a confiabilidade da medida, o SGQ deve considerar a incerteza herdada da calibração do equipamento e somar as incertezas da faixa da curva analítica oriunda do modelo matemático e a incerteza oriunda da preparação das concentrações das soluções padrão da curva analítica. É importante frisar que para garantir a confiabilidade do resultado do ensaio, deve-se conhecer as incertezas do processo como um todo, e que a 
construção da curva analítica ou de calibração é apenas uma das etapas.

O presente artigo visa propor uma discussão acerca da definição das variáveis críticas inerentes à construção da curva analítica e seus controles para dar maior segurança na expressão das medidas e que possam estar em conformidade com as três normas citadas.

\section{Experimental e resultados}

Das três normas referenciadas, a ISO 17025 é a mais restritiva no que se refere à calibração dos equipamentos e à confiabilidade do método ou procedimento utilizado, por tratar dos requisitos gerais para a competência da organização na condução de ensaios e/ou calibração do escopo de interesse. Os elementos da norma estabelecem que o método do ensaio/calibração seja normalizado ou que se deve estabelecer regras para validação do método quando não normalizado ou estiver sendo utilizado fora das condições em que foi validado.

Se o procedimento de validação do método for conduzido seguindo diretrizes ${ }^{[8]}$ e se os experimentos tiverem sido planejados, pode-se utilizar os resultados obtidos para estimar a incerteza da medida. Neste sentido, duas classes de fatores podem influenciar a estimativa da incerteza da medida, os fatores indiretos representados principalmente pela infraestrutura da organização e a qualificação do pessoal; e os fatores diretos representados pela (i) representatividade e integridade da amostra no que tange a retirada e transporte até o local do ensaio e sua manipulação, pelo (ii) método do ensaio, pelas (iii) substâncias ou materiais de referência e pelo (iv) equipamento utilizado na construção da curva analítica.
A confiabilidade da medida inclui: (i) a calibração do instrumento, (ii) a tolerância do ensaio e (iii) a frequência das verificações periódicas. Assim, o que é conhecido como curva de calibração ou curva analítica se constitui para a norma em um conjunto de operações que representam a verificação da manutenção da calibração do instrumento entre períodos de calibração, que é denominado de qualificação instrumental ou conformidade do sistema quando o equipamento é um conjunto de instrumentos. Como as soluções padrão diluídas são preparadas, é necessária a confirmação das operações de preparo delas no estabelecimento da curva de calibração. Assim, é comum utilizar substâncias de referência de diferentes lotes ou fabricantes e utilizar uma delas para estabelecer a curva analítica e outra como amostra de controle. Alternativamente, é recomendada a utilização de amostra-testemunha ou matriz com adição de concentração conhecida, em um lote pré-definido de amostras e submetê-la a todas as etapas do ensaio e os resultados dessa amostra controle são mostrados em carta controle $\mathrm{e}^{[9]}$.

\subsection{Estabelecendo a exatidão da calibração}

No processo analítico, o equipamento responsável pela medida da resposta de interesse deve estar calibrado, isto é, o seu erro em relação a um padrão de referência deve ser estabelecido. O erro obtido, somado à incerteza padrão do procedimento de calibração, resulta na tolerância do equipamento. Como regra, a tolerância do equipamento deve ser menor que a tolerância do processo. $\mathrm{O}$ cálculo da tolerância do equipamento e sua comparação com a tolerância do processo constituem o que nas normas ISO é chamado de análise crítica do certificado de calibração. Já a incerteza padrão do certificado de calibração, a ISO 9001 denomina de incerteza 
herdada. Uma importante consideração, nem sempre seguida, é que o erro utilizado no cálculo deve ser do ponto de calibração mais próximo da medida a ser efetuada.

\subsection{Estabelecendo a precisão da operação em termos da incerteza}

Para a construção da curva de calibração ou analítica, uma série de soluções padrão em concentrações crescentes deve ser preparada para ser medida. Via de regra, para esta operação, é tomada uma massa ou volume da substância de referência e avolumada com um solvente. Posteriormente, são tomadas várias alíquotas dessa solução padrão estoque e diluídas na proporção desejada. As soluções padrão diluídas são então submetidas à medida, geralmente em triplicata. A estimativa da incerteza deve levar em conta a exatidão dos equipamentos utilizados nesta etapa, como balança, recipientes volumétricos, por exemplo, e a precisão da operação de seu uso, como preenchimento do balão volumétrico, tomada de massa.

As concentrações esperadas contra as concentrações obtidas são submetidas a uma equação polinomial para obtenção de um modelo matemático que represente a curva de calibração.

\subsection{Parâmetros para avaliação dos estimadores da curva de calibração ou curva analítica}

Antes da construção da curva é aconselhável aplicar o teste de Grubbs e o teste de Cochran no conjunto de medidas obtidas. Tais testes devem ser aplicados para avaliar a ausência de valores discrepantes para cada nível de concentração e a homocedasticidade dos dados, respectivamente.

Como as concentrações das soluções padrão trazem uma propagação de erros, desde a concentração da substância de referência até a pre- paração das soluções padrão diluídas propriamente ditas, as concentrações esperadas não são exatas. Assim, não é razoável usar interpolação, ou seja, exigir que os resultados satisfaçam exatamente os valores esperados. Desta forma, em vez de recorrer a um polinômio que passe exatamente por todos os pares de valores $\left(x_{i}, f\left(x_{i}\right)\right)$, $i=0, \ldots, n)$, a melhor abordagem será ajustar a função, $g(x)$, o mais próximo possível dos pontos $\left.\left(x_{\mathrm{i}}, f\left(x_{\mathrm{i}}\right)\right), i=0, \ldots, n\right)$. O modelo mais simples que relaciona as variáveis $\mathrm{x}$ (concentração) e y (resposta) é dada pela equação de uma reta expressa pela equação:

$$
y=\beta_{0}+\beta_{1} x
$$

onde $\beta_{0}$ e $\beta_{1}$ são os parâmetros do modelo.

A concentração $x_{i}$ é denominada valor da variável explicativa ou independente e a imagem de $x_{i}$ pela reta $y=\beta_{0}+\beta_{1} x$ é chamado de valor predito, $\hat{y}_{\mathrm{i}}$, e $\mathrm{y}_{\mathrm{i}}$ é o valor da variável resposta ou dependente. A diferença entre $y_{i}$ e $\hat{y}_{i}$ é o desvio representado pela distância vertical do ponto à linha reta. Quando se considera a soma dos quadrados dos desvios, obtém-se uma medida do desvio total dos pontos observados à reta estimada.

Um modo de estimar os coeficientes $\beta_{0}$ e $\beta_{1}$ é determinar o mínimo da função $\mathrm{D}\left(\beta_{0}\right.$ e $\left.\beta_{1}\right)$ em relação a $\beta_{0}$ e $\beta_{1}$. Tal modo é conhecido como método dos mínimos quadrados, uma vez que se quer minimizar uma função quadrática. Assim, a melhor reta usando o método dos mínimos quadrados que melhor se ajusta aos dados do diagrama de dispersão é dada por:

$$
y=a+b x
$$

onde o estimador b, denominado coeficiente angular, representa a inclinação da curva e o estimador a, denominado coeficiente linear, representa a interseção da reta no eixo y. 
O valor dos estimadores a e b são calculados usando as equações a seguir:

$$
\begin{aligned}
& b=\frac{n \sum\left(x_{i} y_{i}\right)-\sum x_{i} \sum y_{i}}{n \sum\left(x_{i}\right)^{2}-\sum x_{i}} \\
& a=\frac{\sum y_{i}-b \sum x_{i}}{n}
\end{aligned}
$$

onde $\mathrm{n}$ representa o número de pontos ou níveis das soluções padrão.

O coeficiente de determinação $\left(R^{2}\right)$ que é uma maneira de medir a qualidade do ajuste linear, é dado por:

$$
R^{2}=\frac{\left(\sum_{i=1}^{n} x_{i} y_{i}-\frac{1}{n} \sum_{i=1}^{n} x_{i} \sum_{i=1}^{n} y_{i}\right)^{2}}{\left(\sum_{i=1}^{n} x_{i}^{2}-\frac{1}{n}\left(\sum_{i=1}^{n} x_{i}\right)^{2}\right)\left(\sum_{i=1}^{n} y_{i}^{2}-\frac{1}{n}\left(\sum_{i=1}^{n} y_{i}\right)^{2}\right)}
$$

com $0 \leq \mathrm{R}^{2} \leq 1$ e quanto mais próximo da unidade, melhor é o ajuste.

Finalmente, os resíduos do modelo são calculados por:

$$
r_{i}=y_{i}-\hat{y}_{i}
$$

\subsubsection{Teste de hipóteses para a curva}

Para garantir que o modelo reduzido pode ser utilizado, ao invés do completo, deve ser provado que o valor do estimador a seja equivalente a zero com grau de confiança estabelecido. O teste de hipótese estabelecido é $\mathrm{H}_{0}: \mathrm{a}=0$ e $\mathrm{H}_{1}: \mathrm{a} \neq 0$.

Para que a $\mathrm{H}_{0}$ seja verdadeira, a Soma Quadrada dos Erros (SQE) deve ser menor ou igual à Soma Quadrada da Regressão (SQR). Caso contrário, rejeita-se $\mathrm{H}_{0}$. As somas quadradas são dadas por:

Soma quadrada total

$$
S Q T=\sum_{i=1}^{n}\left(y_{i}-Y\right)^{2}
$$

Soma quadrada dos erros (resíduos)

$$
S Q E=\sum_{i=1}^{n}\left(y_{i}-\hat{y}_{i}\right)^{2}
$$

Soma quadrada da regressão

$$
S Q R=\sum_{i=1}^{n}\left(\hat{y}_{i}-Y\right)^{2}
$$

\subsubsection{Desvio padrão da curva}

O desvio padrão da curva de calibração ou curva analítica, $D P_{\text {curva }}$, é dada por:

$$
D P_{\text {curva }}=\frac{D P_{x, y}}{b} \sqrt{\frac{1}{p}+\frac{1}{n}+\frac{(Y-\hat{Y})^{2}}{D P_{x, x}}}
$$

onde b é a inclinação da reta, $\mathrm{n}$ representa $\mathrm{o}$ número de níveis da curva, $\mathrm{p}$ o número de repetições da medida, Y é a concentração média esperada e $\hat{Y}$ é a concentração média predita, $D P_{x, y} \mathrm{e}$ $D P_{\mathrm{x}, \mathrm{x}}$ representam os desvios padrão dos desvios dados por:

$$
D P_{y, x}=\sqrt{\frac{\sum\left(y_{i}-\hat{Y}\right)^{2}}{N-2}}
$$

onde $\mathrm{N}=\mathrm{p} \times \mathrm{n}$.

$$
D P_{x, x}=\sum\left(x_{i}-X\right)^{2}
$$

\subsubsection{Estimativa da incerteza da curva}

No cálculo da estimativa da incerteza da curva de calibração ou analítica, é levada em consideração a incerteza padrão associada ao preparo das soluções padrão diluídas e do modelo matemático, sendo possível analisar as contribuições individuais. A incerteza padrão combinada é dada por:

$$
\mu_{\text {processo }}=D P_{\text {curva }}+\mu_{\text {preparo das soluçôes }}
$$




\section{Procedimento operacional padrão}

No Laboratório de Cromatografia - CROMA da USP de São Carlos foi elaborado um procedimento operacional padrão que teve como objetivo estabelecer a contribuição da incerteza da curva analítica ou de calibração sobre a medida do ensaio. A seguir, é descrito e comentado um exemplo de como foi aplicado este procedimento na determinação de fluazuron em tecidos animais por HPLC-MS/MS.

\subsection{Teste de Grubbs}

Foram preparados seis níveis de concentração do analito fluazuron a partir de diluições sucessivas de uma solução padrão estoque obtida da substância de referência. Cada nível de con- centração foi medido em triplicata. Os resultados foram submetidos ao teste de Grubbs e os resultados são mostrados na Tabela 1 .

Os resultados de $\mathrm{G}_{\text {calculado }}$ foram menores que $\mathrm{G}_{\text {crítico' }}$ em todos os casos estudados; portanto, não há valores discrepantes no conjunto. Desta forma, todos os valores devem ser usados.

\subsection{Teste de Cochran}

O mesmo conjunto de dados foi utilizado para o teste de Cochran. Os resultados são mostrados na Tabela 2.

Os resultados de $\mathrm{C}_{\text {calculado }}$ foram menores que $\mathrm{C}_{\text {crítico }}$, em todos os casos estudados e, portanto, as variâncias dos resultados são homogêneas, mostrando a homocedasticidade entre os dados.

Tabela 1 Teste de Grubbs para as medidas das soluções padrão diluídas de fluazuron.

\begin{tabular}{rrrrrrrrr}
\hline & Medida 1 & Medida 2 & Medida 3 & Média & $\begin{array}{r}\text { Desvio } \\
\text { padrão }\end{array}$ & $G_{\text {medidal }}$ & $G_{\text {medida2 }}$ & $G_{\text {medida3 }}$ \\
\hline Nível 1 & 27,0 & 25,5 & 27,3 & 26,6 & 1,0 & 0,415 & $-1,141$ & 0,726 \\
Nível 2 & 55,5 & 56,9 & 57,3 & 56,6 & 1,0 & $-1,134$ & 0,378 & 0,756 \\
Nível 3 & 116,5 & 119,7 & 118,7 & 118,3 & 1,7 & $-1,091$ & 0,873 & 0,218 \\
Nível 4 & 255,5 & 242,1 & 236,6 & 244,7 & 9,7 & 1,107 & $-0,270$ & $-0,837$ \\
Nível 5 & 378,6 & 385,8 & 382,2 & 382,2 & 3,6 & $-1,000$ & 1,000 & 0,000 \\
Nível 6 & 468,8 & 461,6 & 458,0 & 462,8 & 5,5 & 1,091 & $-0,218$ & $-0,873$ \\
\hline
\end{tabular}

Para $n=6$ e $95 \%$ de confiança, o valor máximo permitido de $\mathrm{G}$ é 1,887.

Tabela 2 Teste de Cochran para as medidas das soluções padrão diluídas de fluazuron.

\begin{tabular}{|c|c|c|c|c|c|c|c|}
\hline & Medida 1 & Medida 2 & Medida 3 & Média & $\begin{array}{l}\text { Desvio } \\
\text { padrão }\end{array}$ & Variância & $\mathrm{C}_{\text {calculado }}$ \\
\hline Nível 1 & 27,0 & 25,5 & 27,3 & 26,6 & 1,0 & 0,8 & \multirow{6}{*}{0,5858} \\
\hline Nível 2 & 55,5 & 56,9 & 57,3 & 56,6 & 1,0 & 0,1 & \\
\hline Nível 3 & 116,5 & 119,7 & 118,7 & 118,3 & 1,7 & 0,6 & \\
\hline Nível 4 & 255,5 & 242,1 & 236,6 & 244,7 & 9,7 & 17,2 & \\
\hline Nível 5 & 378,6 & 385,8 & 382,2 & 382,2 & 3,6 & 4,3 & \\
\hline Nível 6 & 468,8 & 461,6 & 458,0 & 462,8 & 5,5 & 6,3 & \\
\hline
\end{tabular}




\subsection{Estimativa da incerteza do preparo das soluções padrão diluídas}

A Figura 1 mostra as etapas críticas do processo de preparação das soluções diluídas, bem como as fontes de incerteza. Para a estimativa da incerteza padrão combinada desta etapa, foi calculada a incerteza padrão do preparo de uma solução padrão estoque, duas diluições subsequentes para atingir um nível de concentração adequado, e outras seis diluições, com fortificação da amostra testemunha nos níveis de concentração da curva analítica.

A equação para a estimativa da incerteza padrão combinada para o preparo das soluções padrão diluídas foi:

$$
\mu_{\text {preparo }}=\underbrace{\left(\frac{\mu_{\text {massa }}}{m}\right)^{2}+\left(\frac{\mu_{\text {dil1 }}}{v_{\text {dil1 }}}\right)^{2}}_{\text {Solução estoque }}+\underbrace{\left(\frac{\mu_{a l 1}}{v_{a l 1}}\right)^{2}+\left(\frac{\mu_{\text {dil2 }}}{v_{\text {dil2 }}}\right)^{2}}_{\text {Diluição 1 }}+\underbrace{\left(\frac{\mu_{a l 2}}{v_{a l 2}}\right)^{2}+\left(\frac{\mu_{\text {dil3 }}}{v_{d i l 3}}\right)^{2}}_{\text {Diluição 2 }}+\underbrace{\sum_{n=1}^{6}\left(\frac{\mu_{a l 3}}{v_{a l n}}\right)^{2}}_{\begin{array}{c}
\text { Níveis da } \\
\text { curva }
\end{array}}
$$

Com os dados obteve-se uma incerteza de 0,002 ng.g ${ }^{-1}$ para a preparação das soluções padrão. É importante destacar que o objetivo do presente trabalho não é o cálculo da estimativa da incerteza para esta etapa. A maneira de obter estes cálculos foi descrita por Meyer ${ }^{[10]}$ e Ratola et al. ${ }^{[11]}$.

\subsection{Cálculo do Desvio Padrão e estimativa da incerteza da Curva Analítica ou de Calibração}

Os estimadores a e b foram calculados a partir do conjunto de medidas obtidas, e a equa-

\section{Pesar 10,1 mg substância de referência}

Completar para volume de $10 \mathrm{~mL}$

Alíquota de $240 \mu \mathrm{L}$ da solução-padrão estoque

Completar para volume de $10 \mathrm{~mL}$

Alíquota de $1250 \mu \mathrm{L}$ da solução-padrão diluída

Completar para volume de $10 \mathrm{~mL}$

Alíquotas para os diferentes níveis de concentração

\section{- Repetibilidade da pesagem}

Exatidão da balança

- Repetibilidade do preenchimento

- Exatidão do balão volumétrico

- Repetibilidade do preenchimento

- Exatidão da micropipeta

Repetibilidade do preenchimento

Exatidão do balão volumétrico

Repetibilidade do preenchimento

Exatidão da micropipeta

Repetibilidade do preenchimento

Exatidão do balão volumétrico

- Repetibilidade do preenchimento

Exatidão do balão volumétrico

Figura 1 Fluxograma das etapas do preparo de solução padrão diluída a partir da substância de referência. 


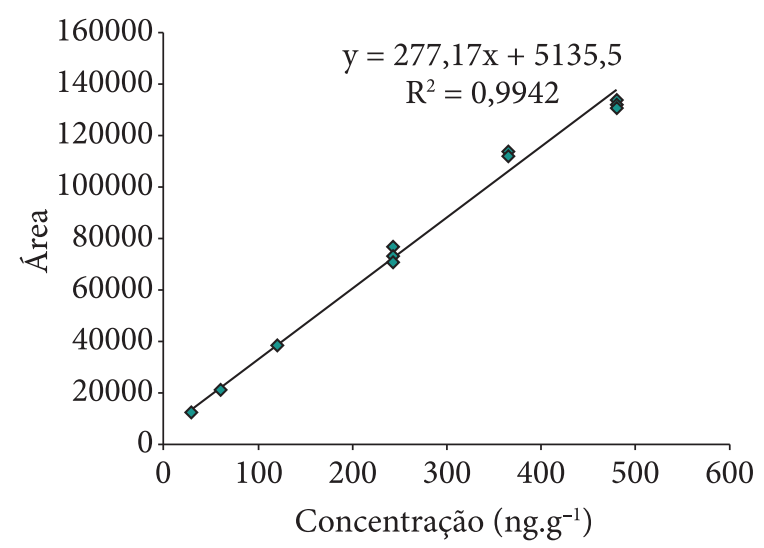

Figura 2 Curva analítica obtida utilizando o conjunto de dados no programa Excel.

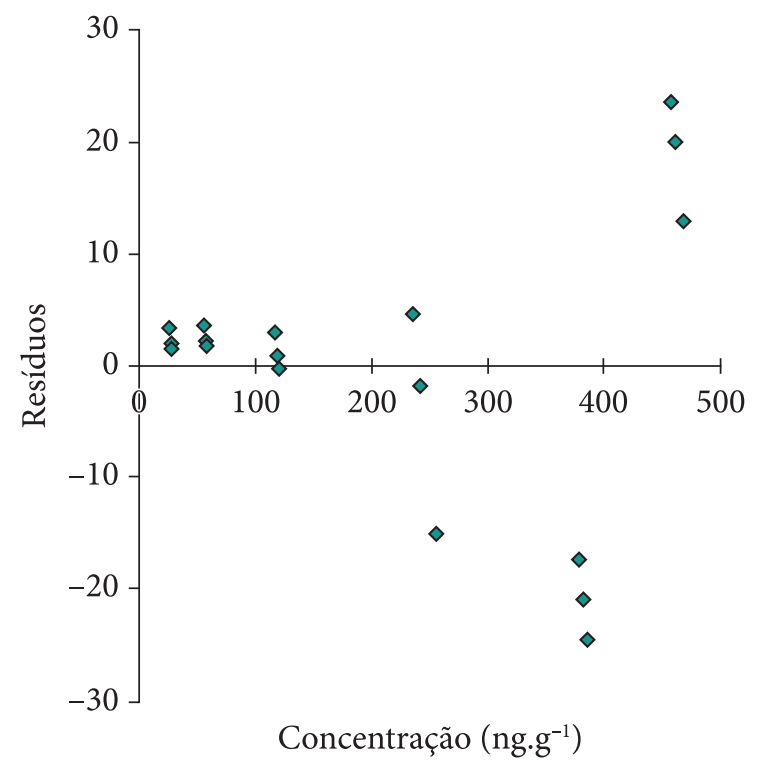

Figura 3 Gráfico de resíduos encontrados entre o valor esperado e o valor predito para cada nível de concentração.

ção da reta resultante é $y=277,17 x+5135,5$. A Figura 2 mostra o gráfico das respostas contra concentração. É importante frisar que os valores dos estimadores determinados pela aplicação das fórmulas foram obtidos no programa Excel.

Com a equação da reta obtida, foram calculadas as concentrações preditas das soluções utilizando suas medidas. Com as soluções preditas calculadas, foi possível estabelecer os resíduos.
É importante frisar que os valores dos resíduos obtidos pela aplicação da fórmula foram similares aos valores obtidos pelo ANOVA do Excel. O gráfico de resíduo é mostrado na Figura 3.

A análise do gráfico de resíduo permite concluir que a variabilidade situa-se próximo de zero e, consequentemente, o que denota um comportamento aleatório dos resíduos e magnitude bastante pequena, reforçando a premissa de que o modelo se ajusta bem para predizer as concentrações de amostras desconhecidas. Contudo, a variabilidade do último nível denota que a resposta do detector utilizado neste ensaio pode estar próxima do limite de saturação do sinal. Neste caso, é aconselhável a construção de duas faixas de concentração para aproveitar adequadamente a melhor capacidade de medida do equipamento usado.

O teste de hipótese foi aplicado ao conjunto de dados e os resultados obtidos foram $\mathrm{SQE}=2797$ e SQR = 480 260. Portanto, a hipótese nula, ou a de que o estimador a é equivalente a zero é válida, pois a soma quadrada dos resíduos ou erros é muito menor que a soma quadrada total, denotando que a equação reduzida pode ser usada.

Usando o módulo de análise de dados, na caixa de dados do programa Excel, e selecionando regressão com os valores de concentração esperada como variável $x$ e os de concentração predita como variável y com significância estatística de 95\%, é possível estabelecer uma nova relação das variáveis e estimar os intervalos de confiança dos estimadores a e b. Os dados são mostrados na Figura 4.

O cálculo do desvio padrão da curva analítica ou de calibração foi determinado utilizando-se as fórmulas descritas anteriormente. Os valores encontrados são mostrados na Tabela 3 . É importante frisar que os valores obtidos pela aplicação da equação foram similares aos valores do ANOVA do Excel. 


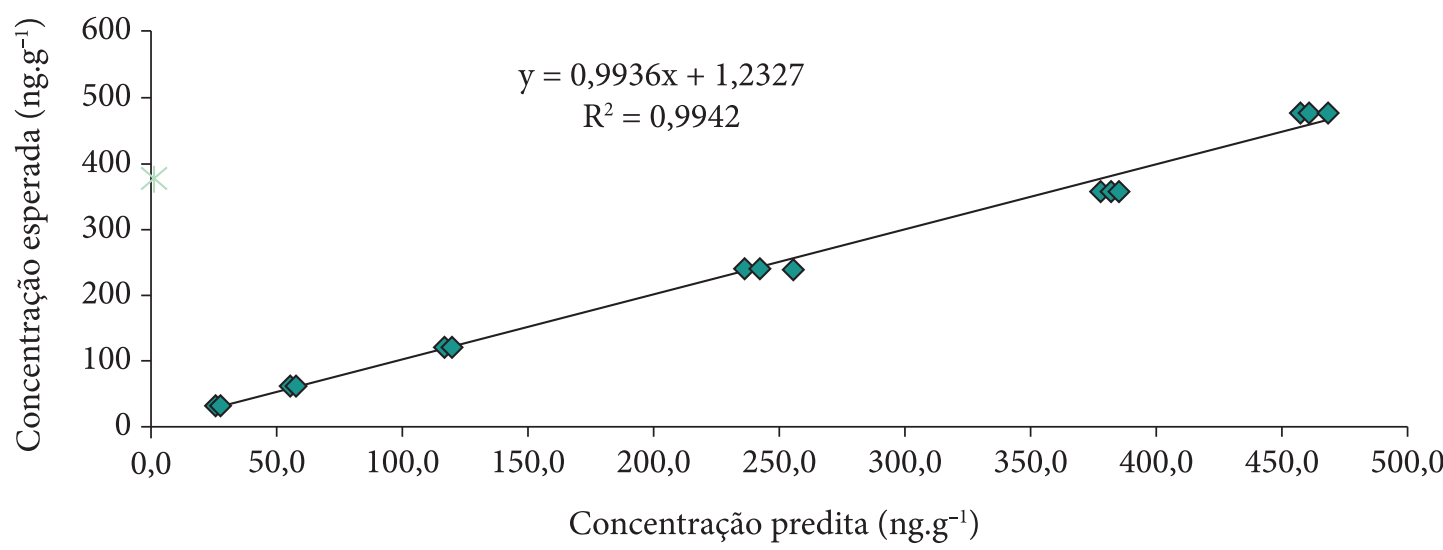

Figura 4 Gráfico obtido através do Excel relacionando as concentrações estimadas e esperadas, com estimadores $\mathbf{a}=\mathbf{1 , 2 3 2 7}$ e $\mathbf{b}=\mathbf{0 , 9 9 3 6}$, com erros padrão de 5,137 e $\mathbf{0 , 0 1 9}$; Intervalos de confiança (95\%) inferior de $-9,6574$ e 0,9532 e superior de $\mathbf{1 2 , 1 2 2 7}$ e $\mathbf{1 , 0 3 3 8}$, respectivamente.

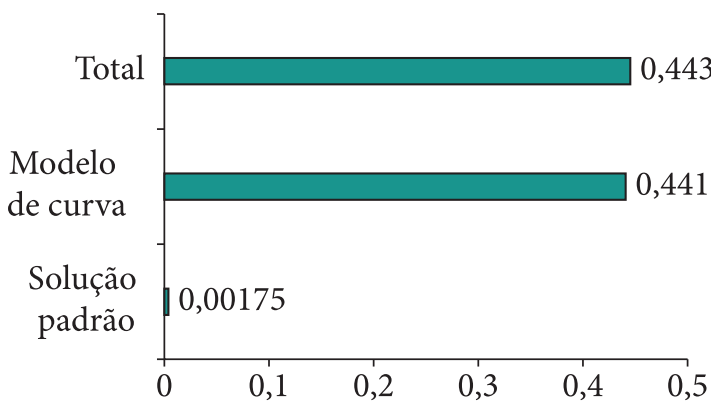

Figura 5 Gráfico de colunas que mostra a incerteza padrão combinada do processo de construção da curva e as contribuições parciais de cada uma das etapas.

Tabela 3 Valores encontrados das variáveis para o cálculo do desvio padrão da reta.

\begin{tabular}{cccccc}
\hline$y$ & $\hat{Y}$ & $D_{x, x}$ & $D_{y, x}$ & $b$ & $D_{\text {curva }}$ \\
\hline 215 & 215 & 477450 & 172,7444 & 277,17 & 0,441 \\
\hline
\end{tabular}

Com os dados, foi possível estabelecer a contribuição do uso do modelo na incerteza da curva analítica ou de calibração. $O$ desvio padrão da curva analítica calculado foi 0,441 ng. $\mathbf{g}^{-1}$.

A incerteza padrão combinada da construção da curva analítica ou de calibração foi feita somando-se a incerteza oriunda do preparo das soluções com a aplicação do modelo de regres- são. A incerteza da curva obtida foi $\mathbf{0 , 4 4 3} \mathbf{n g}^{-\mathrm{g}^{-1}}$, sendo $\mathbf{0 , 4 4 1} \mathbf{n g} \cdot \mathrm{g}^{-1}$ relativa ao modelo da curva e 0,002 ng. g $^{-1}$ da preparação da solução.

Finalmente, foi possível estabelecer a contribuição de cada um dos parâmetros na incerteza combinada do processo. O resultado é mostrado na Figura 5.

\section{Conclusão}

A discussão promovida neste artigo utiliza conceitos de cunho matemático aplicados em ensaios químicos sem, contudo, entrar em detalhes teóricos, abordando mais o contexto prático de como utilizar tais conceitos em conformidade com as diretrizes das normas de gestão da qualidade.

A aplicação dos conceitos estabelecidos por documento orientativo, portarias e legislações em conformidade às diretrizes das normas ISO 17025 , ISO 9001 e BPL visa dar maior confiabilidade e rastreabilidade ao conjunto de dados medidos e ao resultado expresso no relatório de ensaios, aplicando conceitos estatísticos para tomada de decisão e evitando, desta forma, julgamentos subjetivos. Além de consistência, a comparabilidade é o principal objetivo dessa aplicação. 
Neste artigo foi demonstrada a viabilidade do uso dessas orientações em um exemplo prático. Assim, foi possível verificar no exemplo que a contribuição na incerteza padrão combinada do modelo matemático simplificado que representa a curva de calibração é muito mais significativa que a incerteza da etapa de preparo das soluções. Contudo, para cada aplicação, sugere-se que essas contribuições sejam demonstradas, pois os dados utilizados neste trabalho representam apenas uma aplicação específica. Na prática, tem-se observado que, em outras aplicações, a etapa de preparo das soluções tem maior contribuição na incerteza padrão combinada. Por essa razão, recomenda-se que para cada aplicação seja executado procedimento similar para avaliar as contribuições e, consequentemente, ações corretivas que possam minimizar o impacto dessa etapa na incerteza padrão do ensaio como um todo.

\section{Referências}

1 Bahia Filho O. A importância da metrologia química nos resultados das análises de resíduos. Scientia Chromatographica 2009; 1(4):49-54.

2 González FJE, Torres MEH, Frenich AC, Vidal JLM, Campana AMC. Internal quality-control and laboratory-management tool for enhancing the stability of results in pesticide multi-residue analytical methods. Trends in Analytical Chemistry 2004; 23(5):361-369.

3 International Laboratory Accreditation Cooperation ILAC. ILAC-P14:12/2010: ILAC policy for uncertainty in calibration. ILAC, 2010.
4 Associação Brasileira de Normas Técnicas - ABNT. NBR ISO/IEC 17025: Requisitos gerais para a competência de laboratórios de ensaio e calibração. Rio de Janeiro: ABNT, 2005.

5 Associação Brasileira de Normas Técnicas - ABNT. NBR ISO 9001:2008: Sistemas de gestão da qualidade Requisitos. Rio de Janeiro: ABNT, 2009.

6 Instituto Nacional de Metrologia, Normalização e Qualidade Industrial - INMETRO. NIT-DICLA-035: Requisitos gerais para laboratórios segundo os princípios das boas práticas de laboratório (BPL). INMETRO, 2009. Revisão 01.

7 González AG, Herrador MA. A pratical guide to analytical method validation, including measurement uncertainty and accuracy profiles. Trends in Analytical Chemistry 2007; 26(3):227-238.

8 Instituto Nacional de Metrologia, Normalização e Qualidade Industrial - INMETRO. DOQ-CGCRE-008: Orientação sobre validação de métodos analíticos. INMETRO, 2011. Revisão 04.

9 Thompson M, Wood R. Harmonized guidelines for internal quality control in analytical chemistry laboratories (Technical Report). Pure and Applied Chemistry. 1995; 67(4):649-666. http://dx.doi. org/10.1351/pac199567040649

10 Meyer VR. Measurement uncertainty. Journal of Chromatography A 2007; 1158(1-2):15-24. http:// dx.doi.org/10.1016/j.chroma.2007.02.082

11 Ratola N, Santos L, Herbert P, Alves A. Uncertainty associated to the analysis of organochloride pesticides in water by solid-phase microextraction/ gás chromatography-electron capture detection Evaluation using two different approaches. Analytica Chimica Acta 2006; 573-574:202-208. http://dx.doi. org/10.1016/j.aca.2006.03.065

Recebido: 28/07/2011 Aceito: 03/08/2011 